\title{
UNAS NOTAS SOBRE EL CONCEPTO DE LA "MERCANTILIDAD"
}

\author{
JUAN MANUEL MURILLAS ESCUDERO \\ Doctor en Derecho \\ Profesor Asociado del Área de Derecho Civil \\ Universidad de La Rioja
}

SUMARIO

1.- INTRODUCCIÓN. 2.- CONCEPTO DE LA "MERCANTILIDAD”, O SU EQUiVALENTE, "COMERCIALIZACIÓN DEL DERECHO CIVIL". 3.- CONCLUSIONES.

\section{RESUMEN}

Las notas que siguen a continuación pretenden abordar muy sucintamente las relaciones del Derecho Mercantil y el Derecho Civil, a partir del difícil concepto jurídico de la "mercantilidad" y de un sucinto análisis histórico del mismo, sin adoptar ninguna posición previa proclive a ninguna de estas dos ramas del Derecho Privado.

\section{INTRODUCCIÓN}

Son muchas las dificultades de abordar el "carácter de la mercantilidad", debido a la enorme complejidad del problema, cuando además han sido brillantes autores 
quienes se han ocupado de la cuestión, poniendo de relieve esas dificultades para su determinación ${ }^{1}$.

Es evidente que existe una conexión entre el "concepto de Derecho mercantil" y la "mercantilidad", tanto de instituciones concretas como de manifestaciones legislativas, aunque elaborar un concepto de Derecho mercantil constituye una tarea innecesaria para el objetivo que pretendo alcanzar en estas notas ${ }^{2}$, y además no es tema de este estudio determinar ni profundizar en esa doble construcción de la doctrina mercantil respecto al Derecho mercantil en sentido amplio, y el Derecho mercantil en sentido estricto ${ }^{3}$, sino aproximarme al concepto jurídico de la "mercantilidad".

\section{EL CONCEPTO DE LA “MERCANTILIDAD”, O SU EQUIVALENTE, "COMERCIALIZACIÓN DEL DERECHO CIVIL"}

La reflexión parte de la existencia de dos cuerpos legales distintos: un Código de Comercio y un Código Civil regulando unas instituciones y unas relaciones sociales comunes a un campo y al otro, con diferentes criterios ${ }^{4}$, generando una incertidumbre jurídica que en muchos casos no hay certeza de saber cuando se encuentra uno en un campo o en el otro ${ }^{5}$, y es eso precisamente el motivo fundamental que me lleva a reflexionar sobre el concepto de la "mercantilidad" o de la "civilidad", o sus equivalentes más conocidos como "comercialización del Derecho civil" o "civilización del Derecho mercantil ${ }^{6 \prime}$.

La base sobre la que se pretendió construir la "comercialización del Derecho civil", , consiste en una petición de principio. Los primeros pasos del Derecho mercantil

${ }^{1}$ Cfr. Por todos, para no ser exhaustivo en la nota, véase, LANGLE, "El inquietante problema de la homogeneidad y unidad interna del Derecho Mercantil”, en RDM, 80, 1961, pp. 319-377. Más reciente, entre otros, FONT GALÁN, “¿Hacia un sistema jurídico mercantil de “faz completamente nueva?”. La Ley 26/1984, de 19 de julio, para la defensa de los consumidores y usuarios: un instrumento para la realización histórica de un Derecho Mercantil del Estado Social” en RDM, 177, 1985, pp. 381-417.

${ }^{2}$ El concepto de Derecho mercantil ha pasado por numerosas etapas más o menos históricas. No voy a hacer la nota interminable porque la doctrina mercantil ha tratado con profundidad numerosas veces el tema. Basta pensar que en las oposiciones a Cátedra con la anterior Ley de Reforma Universitaria (LRU) en el Proyecto docente e investigador, se debía exponer el concepto de Derecho Mercantil. No obstante, por su significado recojo las palabras de uno de los más insignes mercantilistas: “(...Y entonces, si hacemos esto de abandonar el bosque y remontarnos a la colina, descubriremos en el Derecho mercantil un pasado lógico y armonioso, un presente muy confuso y un porvenir bastante incierto)". Vid. GARRIGUES, "Derecho Mercantil y Derecho Civil", en Anales de la Academia Matritense del Notariado, 15, Madrid, 1967, pp. 431-454, esp. p. 435.

${ }^{3}$ Cfr. VICENT CHULIÁ, Introducción al Derecho Mercantil, 6a edición, Valencia, 1993, pp. 21-26.

${ }^{4}$ Cfr. A. BERCOVITZ, "En torno a la unificación del Derecho Privado", En Estudios Jurídicos en Homenaje al Profesor Federico de Castro, I, pp. 151-168, esp. p. 161.

${ }^{5}$ Véase, GARRIGUES, "Derecho Mercantil ...", cit., pp. 438 y ss.

${ }^{6}$ Muy significativas en este sentido las palabras de GARRIGUES, "En torno a la Reforma del Derecho Mercantil”, en Conferencia de clausura de las Jornadas sobre la Reforma de la Legislación Mercantil, Madrid, 1979, p. 375.

${ }^{7}$ Creo que la idea es bastante clara. La duplicidad de términos, Derecho Especial (igual a Derecho mercantil) y Derecho Común (igual a Derecho civil) giraba sobre una idea, al Derecho Civil o Derecho Común era el Derecho aplicable a todas las personas, mientras que el Derecho Mercantil era un Derecho especial, que se aplicaba a un grupo de personas con un elemento común a todas ellas, la actividad 
se dan con un claro sentido profesional y de clase, o si se prefiere, con unas notas claras de habitualidad y reiteración en la actividad, estando asentado todo criterio de "profesionalidad" por la repetición rutinaria de los mismos actos con distintos sujetos pasivos, de una especie de capacitación proporcionada por lo que llamamos "rutina profesional", la "habituación", pues mientras los sujetos pasivos cambian, el sujeto activo, mercader o comerciante permanece en su inalterabilidad ${ }^{8}$.

La carencia de un poder político tal cual hoy se conoce y el carácter gremial y estamental de la sociedad medieval, crearon las condiciones adecuadas para el nacimiento de una normativa peculiar, donde frente a las normas recién recibidas a través del Corpus Iuris Justinianeo se instalaron unas propias, cuya esencia era la profesionalidad y la asunción del riesgo utilizando el transporte marítimo entre determinados puertos y mercados, frente a la postura estática del viejo Ius Civile de origen romano, y así se va a constituir a lo largo de una lenta evolución histórica el germen del Derecho mercantil, como especialidad ${ }^{9}$ distinta desglosada del viejo seno del ius privatum ${ }^{10}$.

Cuando en el siglo XIX llega la etapa codificadora va a cristalizar la situación mediante la instauración de una dualidad: un Código de Comercio y un Código Civil, aunque la acusada tendencia internacionalista del comercio, haga más fácil la consecución de la codificación mercantil, que ya tenía una larga serie de manifestaciones previas de carácter privado, frente al Código Civil, más polémico debido a las peculiaridades locales y a los problemas políticos de todos conocidos, principalmente la resistencia a conservar el Derecho foral o especial de determinadas regiones ${ }^{11}$.

comercial que desarrollaban, esto es, eran comerciantes, siendo su nota más característica la "habitualidad" de una conducta y esta pronto se transforma en "profesionalidad".

${ }^{8}$ La concepción del Derecho Mercantil como especialidad de una "clase" profesional y sociológica, basada más en la habitualidad y en lo que se podrían llamar "estereotipos del buen hacer comercial", es la idea genuina del Derecho Mercantil, encontrándose ya constantemente en toda la doctrina mercantil, desde el ya muy lejano BENITO Y ENDARA, quien da a la imprenta sus Estudios de Introducción al Derecho Mercantil, en Valencia en el año 1888.

${ }^{9}$ Ha sido muy bien sintetizada la idea de especialidad por Bustos Pueche, dice: "Han ido naciendo, así, ramas especiales o subdivisiones del Derecho, que reciben esa calificación de especialidad, no por una razón ontológica, sino por relación a la situación normativa inicial. En la medida en que de un bloque normativo preexistente se desgajó otro subconjunto regulador, para ordenar una parcela de la realidad social que antes se organizaba por aquel bloque primitivo, aparece la rama especial, especial respecto de aquél, que por ello retiene la consideración de Derecho común". Véase, BUSTOS PUECHE, "Sugerencias para reflexionar sobre el fenómeno de la especialización normativa", $A D C, 51,1998,3$, pp. 10711103 .

${ }^{10}$ He consultado todas las "obras históricas" de Derecho Mercantil que he podido conseguir, y sin embargo, no he encontrado quien ha sido el primer autor en aducir la inadecuación del viejo Derecho Civil a las necesidades de la práctica comercial de los profesionales. No obstante, puede ser de interés y ofrecer alguna luz en este sentido, OLIVENCIA RUIZ, "Concepto del Derecho Mercantil”, en Lecciones de Derecho Mercantil, volumen colectivo bajo la coordinación de Guillermo Jesús Jiménez Sánchez, $2^{\text {a }}$ edición, Madrid, 1993, pp. 39-48, esp. pp. 39-41.

${ }^{11}$ Cfr. LACRUZ, Los Derechos Forales, en, Universidad, 1948, pp. 3-17 y de nuevo en, Estudios de Derecho Privado Común y Foral, I, Madrid, 1992, pp. 27-37, esp. p. 36. Problemas que han sido analizados recientemente por, MARÍA REPÁRAZ PADRÓS, en "La crítica contemporánea al Proyecto de Código Civil de 1851", ADC, 50, 3, 1997, pp. 1015-1220. Conviene señalar que así como en Francia bajo el liderazgo de Napoleón se pudo unificar el Derecho civil escrito y el Derecho consuetudinario, aquí en España, no se pudo unificar el Derecho de Castilla y los diversos Derechos Forales. 
Cuando se cae en la cuenta de la existencia del acto de comercio "aislado", o más bien, visto desde la realidad práctica y no desde la dogmática o desde la tópica ${ }^{12}$, del innegable dato de la realización de actos de comercio por quienes no eran habitualmente comerciantes, surge la pretensión de aplicar las normas profesionales comerciales, residuo del corporativismo gremial medieval, a quien no era habitualmente comerciante, y así se acuña el término de "acto mixto", es decir, los realizados entre un comerciante y una persona no comerciante ${ }^{13}$, para dar después origen a la construcción del "acto objetivo de comercio", con la consecuencia que incluso al día de hoy, existen grandes dificultades en la más autorizada doctrina mercantil para precisar su significado, y este fenómeno, se contrapone directamente con el concepto tradicional del Derecho mercantil como Derecho profesional o de clase, dando lugar actualmente a que una determinada parte de la doctrina mercantil se encuentre proclive a retornar al carácter profesional originario ${ }^{14}$.

En España, en el momento actual existen dos normativas distintas y concurrentes presentes en el Código Civil y en el Código de Comercio, basta como ejemplo detenerse a observar el Título IV del Libro I del Código de Comercio para darse cuenta a primera vista de una serie de figuras jurídicas que también tienen su acomodo en el Código Civil ${ }^{15}$, así como determinados contratos presentes en ambos códigos, compraventa, permuta, depósito, fianza ..., entre otros. Su posible explicación se debe a la existencia de un doble dato de carácter exclusivamente anecdótico:

La posibilidad de promulgar un Código de Comercio, no estaba afectado por las resistencias para conseguir un Código Civil.

$\mathrm{Al}$ redactarse posteriormente el Código Civil, no se plantea dogmática ni políticamente, la asunción de la normativa fraccionaria contenida en el Código de Comercio, por ser en ese momento muy clara la existencia de una profesión habitual, peculiar y exclusiva, constituida por el ejercicio del comercio. Pero como los comerciantes habituales no solamente contrataban entre sí, surge la imperiosa necesidad de precisar la normativa aplicable a los llamados actos mixtos, produciéndose una primera e importante expansión del ámbito de aplicación del Código de Comercio.

En este sentido creo que es necesario la conveniencia de resaltar la absoluta sinonimia señalada entre las dos voces "comercial" y "mercantil" en el diccionario de sinóni-

${ }^{12}$ Cfr. TheOdor VIEHWEG, Tópica y Jurisprudencia, traducción de Luis Díez-Picazo, Madrid, 1963, pp. 125-143, esp. pp. 131 y siguientes.

${ }^{13}$ Definidos por ROJO Y FERNÁNDEZ-RÍO, como "aquellos que ofrecen los requisitos del acto de comercio para una parte y no, en cambio, para la otra. Precisamente el ejemplo que suele citarse para clarificar la noción es el de la compraventa para el consumo, que es acto mercantil para el revendedor y civil para el comprador". Vid. La responsabilidad civil del fabricante, Bolonia, 1974, p. 261.

${ }^{14}$ Cfr. PUENTE MUÑOZ, "Notas para un estudio sobre el concepto de Derecho Mercantil", en Homenaje a Joaquin Garrigues, III, Madrid, 1971, pp. 77-85, esp. p. 83.

${ }^{15}$ De un lado hay artículos que apenas difieren, tal como los artículos 51 y 52 (forma de los contratos), 57 (buena fe), 59 (favor debitoris del deudor), 60 (cómputo de días), 61 y 62 (cumplimiento de las obligaciones), y 63 (la mora); y de otra parte, aquellos artículos en donde difiere su contenido, resulta la regulación ofrecida al compararla con la del Código Civil, totalmente dispar y opuesta, solamente en tres casos concretos, artículo 54 (contrato entre personas distantes), 56 (cláusula penal), y los artículos 2 y 50, contrarios entre sí, pese a referirse ambos al Derecho Común, y contrarios al valor y carácter del Título Preliminar del Código Civil, y especialmente al art. 4.3 del mismo texto legal. 
mos de GILI GAYA ${ }^{16}$, pero es posible que sea esa pretendida sinonimia absoluta la que pueda explicar sin necesidad de otros argumentos, la discrepancia en el modo de designar a la especialidad en Francia, Droit Commercial ${ }^{17}$, y en Italia, Diritto Commerciale ${ }^{18}$, mientras en Alemania con el término Handeslrecht ${ }^{19}$, se cubre esa necesidad con una única expresión comprensiva de ambos modismos.

Es cierto que tanto en Francia como en Italia se usa únicamente una expresión, pero el problema planteado es averiguar por qué habiendo sido usada inicialmente una denominación, -Derecho del comercio-, se abandona la misma, pese a la influencia principalmente francesa y en menor medida también italiana en alguno de nuestros textos legales, sin plantearse la transformación del término, tal como se ha justificado y aludido con reiteración al abandono de la denominación "Derecho social" para designar al "Derecho del trabajo" 20 , situación notablemente próxima si se toma en cuenta que constituyen tanto el Derecho mercantil como el Derecho del trabajo, dos nuevas disciplinas que se desglosan del ámbito del Derecho civil y con el cual siguen manteniendo importantes nexos de unión.

Aunque a modo de mera curiosidad intelectual, me hubiera gustado encontrar los argumentos por los que se opta en España por la expresión "Derecho mercantil", separándose del pensamiento francés e italiano, término usado por primera vez por EUGENIO DE TAPIA $^{21}$ en el ya lejano año de 1829 , y aunque es usual en todos los manuales, tratados, y artículos de revistas especializadas, ocuparse del origen histórico del Derecho mercantil ${ }^{22}$,

${ }^{16}$ Cfr. SAmuel GILI GAYA, Vox. Diccionario de Sinónimos, Barcelona, 1958, p. 91. Existe otra edición sin autor absolutamente idéntica, que figura como Diccionario VOX Esencial de la Lengua Española. Sinónimos y Antónimos, Barcelona, 1995, p. 103.

${ }^{17}$ Véase la obra clásica de HenRI HUVELIN, L’Historie du Droit Commercial (Conception generale. Etat actuel des etudes), París, 1904.

${ }^{18}$ A modo de ejemplo véase la obra de GiUSEPPE AULETTA y Nicoló SALANITRO, Diritto Commerciale, Milán, 1994, recientemente editada su $9^{\text {a }}$ edición.

${ }^{19}$ Véase la obra clásica de CLAUS-WILHEM CANARIS, Handelsrecht, Múnich, 1995, editada recientemente su $22^{\mathrm{a}}$ edición.

${ }^{20}$ Para ilustrar en plan paralelo la transformación sufrida en España por la denominación inicial "Derecho del Comercio" para ser sustituida muy pronto por "Derecho Mercantil", creo que es muy expresivo mencionar cómo CASTÁN TOBEÑAS publica un artículo sobre "El Derecho Social" en la $R G L J$, del primer semestre del año 1941, pp. 513 y ss., POLO, escribe en la RDP del mismo año 1941 sobre, "Del contrato a la relación de trabajo", pp. 10-17 y 80-95, y la doctrina laboral, con la única excepción de MENÉNDEZ PIDAL, quien aun en 1952 publica un Derecho Social Español, en dos volúmenes, sin embargo todos los tratadistas restantes desde 1944 usan exclusivamente el término "Derecho del Trabajo", explicando en sus obras las razones de su preferencia por este término.

${ }^{21}$ Cfr. DE TAPIA, Elementos de Jurisprudencia Mercantil, Madrid, 1829.

${ }^{22}$ En este sentido pueden aducirse: ALVAREZ DEL MANZANO Y ALVAREZ DE RIVERA, BONILLA Y SAN MARTÍN, y MIÑANA Y VILLAGRASA, Tratado de Derecho Mercantil, 2 tomos, I, Madrid, 1915, pp. 58-135. ARANDA GONDRA, Orientaciones modernas de la legislación mercantil, Madrid, 1987. ASCARELLI, Iniciación al estudio del Derecho Mercantil, traducción de Evelio Verdera y Tuells, Barcelona, 1964, pp. 29-74. BENITO Y ENDARA, Estudios de Introducción al Derecho Mercantil, Valencia, 1888; Lecciones de Derecho Mercantil, Madrid, 1889, Las bases del Derecho Mercantil, (De la colección de "Manuales Soler"), Madrid, 1909; Manual de Derecho Mercantil, I, Parte General, II, Parte Especial, (Obligaciones mercantiles) Contratos fundamentales, $3^{\text {a }}$ edición, Madrid, 1924. JOSÉ DE BENITO, "Prólogo" a la traducción de la $5^{\text {a }}$ edición italiana en La unidad del Derecho Privado en materia de obligaciones, de César Vivante, 105 páginas, Madrid, 1925; El Derecho Mercantil Español en el siglo XVII, Madrid, 1935. A. BERCOVITZ, "Origen histórico del Derecho Mercantil", en Estudios Jurídicos en Homenaje a Joaquín Garrigues, I, Madrid, 1971, pp. 1-44, esp. pp. 32-44. BOIX RASPALL, "Derecho Mercantil", en Nueva Enciclopedia Jurídica Seix, Barcelona, 1950, pp. 693-724, esp. pp. 711-717. BROSETA, "Concepto sustancial del Derecho Mercantil”, en Studi in memoria di Tullio Ascarelli, IV, Milán, 1969, pp. 2706 y ss.; Manual de Derecho Mercantil, 9a edición, Madrid, 1991. 
en ninguno de ellos se menciona la discrepancia en la denominación existente entraña y los países de nuestro entorno ${ }^{23}$. Toda esta problemática planteada, tiene una manifestación clara en la pretensión de encontrar el concepto de "acto de comercio objetivo"24, así como su consecuencia, que no es otra, que al aplicar por extensión a los no comerciantes la normativa mercantil cuando contratan con un comerciante, conlleva la progresiva disminución del carácter de su especialidad, bien al generalizarse ${ }^{25}$, o bien, por el camino de la disolu-

CALVO ALFAGEME, Apuntes de Derecho Mercantil, 6 ${ }^{\mathrm{a}}$ edición, Valencia, 1960. VICENTE DEL VALlE y MANUEla ZARAGOZA, Derecho Civil y Derecho Mercantil, Madrid, 1994, p. 103. ESPEJO DE HINOJOSA, Curso de Derecho Mercantil, 9a edición, Barcelona, 1935, pp. 29-51, esp. pp. 39-51. FERNÁNDEZ RUIZ, Fundamentos de Derecho Mercantil, Madrid, 1991, pp. 3-12. GARRIGUES, "Setenta y cinco años de Derecho Mercantil", en Homenaje al Profesor Antonio Polo, Madrid, 1981, pp. 263279; "Concepto del Derecho Mercantil”, en RDP, 1943, pp. 369-383; Instituciones de Derecho Mercantil, $2^{a}$ edición, Madrid, 1948, pp. 1-18; "La crisis del Derecho Mercantil Contemporáneo", en Hacia un nuevo Derecho Mercantil. Escritos, lecciones y conferencias, Madrid, 1971, pp. 201-226, esp. p. 212. GIRÓN TENA, "El concepto de Derecho Mercantil: desenvolvimiento histórico y Derecho Comparado", en $A D C$, 7, 1954, 3, pp. 695-807. GÓMEZ SEGADE, “Algunos problemas actuales del Derecho Mercantil”, en Homenaje a Rodrigo Uría, Madrid, 1978, pp. 209-230. GONDRA ROMERO, Derecho Mercantil I. Introducción, Madrid, 1992. JIMÉNEZ DE PARGA, "Condicionamientos históricos del Derecho Mercantil en la fase inicial de su formación", en Homenaje a Rodrigo Uría, Madrid, 1978, pp. 315-333. LANGLE, Manual del Derecho Mercantil Español, Barcelona, 1950, pp. 101-218. LÓPEZ SANZ, Curso de Derecho Mercantil, 4a edición, Valencia, 1991, pp. 11-20. MARTÍ DE EIXALÁ, Instituciones del Derecho Mercantil de España, Madrid, 1848. MOSSA, Historia del Derecho Mercantil en los siglos XIX y XX, traduce Francisco Hernández Borondo, Madrid, 1948. MUÑOZ PLANAS, "La objetivación del Derecho Mercantil en la Codificación Napoleónica", en Homenaje al Profesor Manuel Broseta Pont, II, Valencia, 1995, pp. 2541-2570. OLIVENCIA RUIZ, “Concepto del Derecho Mercantil”, en Lecciones de Derecho Mercantil, cit., pp. 37-46; "El Derecho Mercantil. Origen y evolución histórica”, en Derecho Mercantil, coordinación de Guillermo Jesús Jiménez Sánchez, Barcelona, 1990, pp. 3-46. PASCUAL QUINTANA, “Aspecto histórico del Derecho Mercantil", en RDM, 69, 1958, pp. 7-38. POLO, "El concepto y los problemas del Derecho Mercantil en la legislación y jurisprudencia españolas", en RDP, pp. 1175-1191 y 1957, pp. 3-28. PUENTE MUÑOZ, "Notas para un estudio sobre el concepto de Derecho Mercantil", en Homenaje a Joaquín Garrigues, Madrid, 1971, pp. 75-85. REHME, Historia Universal del Derecho Mercantil, traduce Emilio Gómez Orbaneja, Madrid, 1941. ROCCO, Principios de Derecho Mercantil, $10^{a}$ edición, traduce Revista de Derecho Privado, Madrid, 1981, pp. 5-33. RUBIO, Introducción al Derecho Mercantil, Barcelona, 1969, pp. 5-240; "Sobre el concepto del Derecho Mercantil", en RDM, 12, 1947, pp. 317-380. RUIZ DE VELASCO Y DEL VALLE, Manual de Derecho Mercantil, Bilbao, 1992, pp. 27-43. SANCHEZ CALERO, Instituciones de Derecho Mercantil, 15 a edición, Madrid, 1991. SANTOS MARTÍNEZ, "Sobre los conceptos, sistema y principios del Derecho Mercantil”, en Honor al Profesor Batlle Vázquez, Madrid, 1978, pp. 739-762; Para una didáctica del Derecho con especiales referencias al Derecho Mercantil, Murcia, 1978. SUAREZ-LLANOS GÓMEZ, "Crítica del saber de los mercantilistas", en Homenaje al Profesor Antonio Polo, Madrid, 1981, pp. 1141-1152. URÍA, Derecho Mercantil, 23 a edición, Madrid, 1996. GIUSEPPE VALERI, "Autonomia e limiti del nuovo Diritto Commerciale”, en Rivista di Diritto Commerciale, 1943, I, pp. 22 y ss.; VICENT CHULIÁ, Introducción al Derecho Mercantil, 9a edición, Valencia, 1996; "Doctrina, Ciencia de la Legislación e Institucionalización del Derecho Mercantil en la última década", en Homenaje al Profesor Manuel Broseta Pont, III, Valencia, 1995, pp. 4043-4121; "El Derecho Mercantil del Neocapitalismo", en RDM, 139, 1976, pp. 769. VICENTE Y GELLA, Curso de Derecho Mercantil Comparado, 2 volúmenes, Zaragoza, 1944-1945.

${ }^{23}$ Aunque no he obtenido respuesta a la pregunta que planteo en el texto, puede dar alguna luz al respecto el trabajo de CASTÁN VÁZQUEZ, "La recepción de voces de Derecho Mercantil en el Diccionario de Autoridades", publicado en Homenaje al Profesor Aurelio Menéndez, I, Madrid, 1996, pp. 213225.

${ }^{24}$ Las dificultades para construir el acto de comercio objetivo han sido reconocidas por la generalidad de la doctrina mercantil. Por todos, véase, GARRIGUES, Instituciones ..., op. cit., p. 55. También del mismo autor la afirmación contenida en la "Conferencia de clausura" en las Jornadas sobre la Reforma de la Legislación Mercantil, Madrid, 1979, p. 373.

${ }^{25}$ Cfr. GARRIGUES, "Setenta y cinco años de Derecho Mercantil”, cit., p. 268. 
ción por fragmentación ${ }^{26}$ en otras tantas especialidades nuevas, las cuales naturalmente también reclaman autonomía doctrinal, científica y legislativa ${ }^{27}$, apoyadas sin duda en el momento actual por la preponderancia y la tendencia de proliferación de las leyes especiales frente a los textos legales $\operatorname{codificados}^{28}$; que además éstos en muchas ocasiones no se tienen en cuenta a pesar de la relevancia que siguen conservando ${ }^{29}$, circunstancia ésta puesta de manifiesto de forma precisa por una parte de la doctrina italiana cuando habla del tránsito del monosistema al polisistema ${ }^{30}$.

Sin embargo, esta incertidubre no es nueva, está ya anunciada hace bastante tiempo por GARRIGUES ${ }^{31}$, concretamente desde el año 1948, quizá en términos que el paso del tiempo ha demostrado excesivamente pesimistas, pero con una gran clarividencia de los acontecimientos manifestó que podrá llegar a disolverse la especialidad por absorción, pero eso no supondrá "su muerte" sino su gloria y pervivencia como tal, aunque sea con otro nombre, por cuanto hay necesidades permanentes a las cuales es preciso atender, aunque es evidente que no haya garantía alguna acerca de la perdurabilidad de las especialidades ni de las concepciones heredadas para afrontarlas ${ }^{32}$.

\section{CONCLUSIONES}

En una sociedad tan compleja como es la actual, con una aceleración de los acontecimientos sociales y económicos que no ha tenido parangón en toda la historia de la humanidad, -y, que no se parece en nada a aquella sociedad medieval de artesanos, gremios ..., en la cual se estaban dando los primeros pasos del futuro Derecho mercantil-, es difícil delimitar con nitidez unas señas de identidad precisas de lo "mercantil" o de la "mercantilidad"33, por ello, creo que actualmente debemos centrar nuestros esfuerzos sobre otros ángulos distintos, potenciando precisamente unas líneas de acercamiento entre ambas materias, sin menoscabo de una a costa de la otra, que propició que en el año 1959 se impartiera en Madrid una conferencia con el beligerante título, "Invasión del ámbito civil por las normas mercantiles" 34 , plasmada posteriormente en los "Anales del Notariado", aunque creo que la sensación actual tanto en la doctrina civil como en la doctrina mercantil, hace que ese título hoy se nos aparezca ya como algo superado, porque ni la doctrina civil ni la mercantil van por ese camino.

\footnotetext{
${ }^{26}$ Cfr. LANGLE, “El inquietante problema ...”, op. cit., pp. 319-377, esp. p. 319.

${ }^{27}$ Quizá se está perdiendo una visión de síntesis y del sistema, nada más citar, entre otras, las nuevas especialidades que están surgiendo, el "Derecho del Consumo", "Derecho de los Consumidores y Usuarios", "Derecho de Sociedades", "Derecho de la Competencia y Distribución” (...).

${ }^{28}$ Vid. LÓPEZ y LÓPEZ, "Constitución, Código y Leyes Especiales. En torno a la llamada descodificación”, en Academia Sevillana del Notariado, IV, Madrid, 1991, pp. 451-472, esp. p. 472. DÍEZPICAZO, “Codificación, descodificación y recodificación”, en ADC, 45, 1992, 2, pp. 473-484.

${ }^{29}$ Cfr. GIORGIANNI, "Tramonto della Codificazione. La morte del Codice ottocentesco", en Rivista di Diritto Civile, 26, 1980, 1, I, pp. 52-55.

${ }^{30}$ Expresiones de IRTI, véase, "Leggi speciali”, en Rivista di Diritto Civile, I, 1979, pp. 141 y ss. y el clásico de este autor, La edad de la descodificación, traducción de Luis Rojo Ajuria, Barcelona, 1992, pp. 97-99.

${ }^{31}$ Vid. GARRIGUES, Instituciones de Derecho Mercantil, $2^{\mathrm{a}}$ edición, Madrid, 1948, p. 21.

${ }^{32}$ Cfr. GARRIGUES, "Derecho Mercantil y Derecho Civil”, op. cit., p. 444.

${ }^{33}$ Cfr. GARRIGUES, "Qué es y qué debe ser el Derecho Mercantil”, cit., p. 55.

${ }^{34} \mathrm{Vid}$. FIGA FAURA, "Invasión del ámbito civil por las normas mercantiles", en Anales de la Academia Matritense del Notariado, 28, 1985, pp. 173-192, esp. p. 189.
} 
En este sentido además, se ha visto cómo la unificación legislativa hecha por el Código civil italiano ${ }^{35}$ no ha afectado en Italia ni a la autonomía, ni al propio ser de lo "comercial", aunque en los momentos iniciales hubiera por parte de ambos sectores dudas razonables, más o menos fundadas, en torno al desarrollo de la nueva situación ${ }^{36}$. Por tanto, este acercamiento entre ambas especialidades que no se vea como un intento de comercializar el Derecho civil, y tampoco tratar de civilizar, -lo cual aún tiene connotaciones más peyorativas- el Derecho mercantil.

Entiendo que es más sencillo que todo eso, porque se trata de todo lo contrario, en vez de exagerar elevando las señas de identidad de la especialidad del Derecho mercantil frente al Derecho civil, reconocer y promover un grado de aproximación entre estas dos materias de Derecho Privado llamadas a coexistir y a conservarse, en el que desaparezcan infundadas divergencias y discrepancias, porque además hoy se es plenamente consciente ${ }^{37}$ de cuan poco afecta a la autonomía e independencia del Derecho mercantil el seguir por ese camino, y sin embargo, cuantos beneficios reporta a la claridad, a la seguridad y al perfeccionamiento de nuestro Ordenamiento jurídico, el posibilitar un acercamiento ${ }^{38}$ de ambas materias; y siendo conscientes del tiempo que ha transcurrido desde la promulgación de ambos Códigos, proponer una modernización, en donde, al mismo tiempo que vuelva el Derecho mercantil al seno de su origen profesional, se suprima la necesidad de precisar cual es el verdadero carácter de la mercantilidad, y simultáneamente, se vaya limitando la expansión de un concepto en aras de un principio de justicia ${ }^{39}$, firmemente asentado como uno de nuestros principios constitucionales $^{40}$, consiguiendo a la vez una adecuación del Ordenamiento jurídico a los signos de los tiempos actuales en que debe aplicarse, tal como en el año 1974 propugnaba nuestro Código Civil en el apartado 1 del artículo $3^{41}$, acercando y unificando poco a poco, todo aquello que sea posible de las dos especialidades de forma adecuada y prudente, buscando en el Derecho positivo aquélla solución que sea más justa y demande la sociedad actual, independiente del origen y ubicación de la norma, eliminando los futuros autores de estas propuestas estereotipos doctrinales de especialiad o de escuela ${ }^{42}$.

${ }^{35}$ La misma línea unificadora ha seguido el nuevo Código civil brasileño de 2001.

${ }^{36}$ Véase las significativas palabras de VIVANTE en el primer parágrafo del primer tomo de su Tratatto di Diritto Commerciale, Milán, 1934, pp. 1-25.

${ }^{37}$ Cfr. VICENT CHULIÁ, Introducción al Derecho Mercantil, op. cit., pp. 22-24.

${ }^{38}$ Cfr. A. BERCOVITZ, “En torno a la unificación ...”, cit., p. 152.

${ }^{39}$ Para Vivante constituye un problema de justicia la aplicación de un "derecho de clase", el derecho comercial, a quien no es comerciante, si bien matiza, que como profesor debería sentirse feliz de esa difusión a extraños de la disciplina, como ciudadano considera perturbador que una legislación de clase perjudique a la solidaridad social que debería presidir todos los designios del legislador. Cfr. VIVANTE, Trattato di Diritto Commerciale, I, Milán, 1934, p. 13.

${ }^{40}$ Vid. Art. 9.3 de la Constitución.

${ }^{41}$ Cfr. SALVADOR CODERCH, "Comentario al artículo 3.1 del Código civil”, en Comentario del Código Civil, I, Madrid, 1991, dirigido por Paz-Ares, Díez-Picazo, R. Bercovitz y Pablo Salvador Coderch.

${ }^{42}$ Cfr. VICENT CHULIÁ, Introducción al Derecho Mercantil, op. cit., pp. 21 y ss., con el cual comparto en este tema sus ideas, aunque habla de un sentido "estricto" de Derecho Mercantil y de otro sentido "amplio" del Derecho MercantiL, sin precisar en qué terreno se quiere situar al Derecho Mercantil, aunque termina por decir que en vista de la generalización y aceptación se produce una unificación de hecho y deja de ser una categoría histórica. 